we may add that where the issues to the public are so important as are those which the ophthalmologists have rightly claimed them to be, it is the duty of the Council to concede the introduction of a reform for which such urgent necessity has been shown.

\title{
The Early Treatment of Ophthalmia Neonatorum
}

In our last issue we noted that a practitioner was fined for failing to notify a case of ophthalmia neonatorum. The object of notification is not to prevent the spread of the disease, but to see that the child receives prompt and proper curative treatment, which as a rule cannot be carried out in the patient's own home.

In London, and in several of the larger towns, following the example of Manchester, special hospital accommodation is provided which will receive both mother and baby for treatment. In the metropolis, under the auspices of the Metropolitan Asylums Board, St. Margaret's Hospital, Leighton Road, Kentish Town, was opened last September, and it is proposed at a later date to open another hospital on the south side of the river. Ambulances are provided to remove both mother and baby to the hospital immediately on receipt of a message either by tèlephone or other means of communication with the Metropolitan Asylums Board.

For these hospitals to serve the purpose for which they are intended, namely, the prevention of blindness by corneal ulceration, which usually starts during the first few days of the disease, it is obvious that the cases must be admitted at the earliest possible moment. We are informed that during the first few months in which St. Margaret's Hospital was opened, the average date of admission was three weeks, which is obviously too late to prevent corneal ulceration. Latterly, the conditions have improved, partly on account of the more active representation of the Medical Officers of Health, and partly owing to the fact that the cases are now admitted on the request of the Midwives of the London County Council without losing time in obtaining a certificate from a Medical Officer. In spite of this there are still a number of children admitted to this hospital with severe corneal ulceration in a late stage of the disease. Many of these come from hospitals and general practitioners, both of which, although they have been informed of the existence of special hospitals, have forgotten their existence until too late to save the eyesight of the child. We cannot urge too strongly the necessity of the efficient and early treatment of the disease, and that practitioners and resident medical officers should from time to time be reminded of the existence of special hospitals for its treatment. 